\title{
Algorithm for the Mass-Loss Rate of a Burning Wall
}

\author{
HENRI E. MITLER \\ Center for Fire Research \\ National Bureau of Standards \\ Gaithersburg, Maryland 20899, USA
}

ABSTRACT

A derivation is given for a simple algorithm which yields the quasisteady burning rate of a vertical panel of non-charring, non-melting material in an enclosure with stratification of temperature and oxygen concentration. The algorithm requires the solution of a transcendental equation. Among the thermophysical data which are needed, are the mean flame temperature and the height-dependent absorption coefficient, $\kappa(z)$. It is found from experiment that $\kappa(z)$ is well described for PMMA by a twoparameter expression linear in $1 / z$. Comparison with a transient experiment yields good agreement for the mass-loss rate, over much of the range.

\section{INTRODUCTION}

There are a number of compartment fire models in existence now (see, for example, [1-6]). Most of these lack an appropriate algorithm to describe a wall fire. Of the above, only Smith's model [4] considers wall fires, and it is tied directly to experimental output from a particular apparatus. A general wall-fire model for burning in compartments must be able to predict the ignition, and then the time-dependent burning and spread-rates (upward, lateral, and downward) of a wall fire under conditions of temperature and oxygen concentration stratification. It should include the effects of external radiation, and of melting, charring, and burnthrough. The present work is a step in that direction. So far, there have been three published models for the burning rate of turbulent wall fires [79]; several for upward spread $[10-16]$.

The problem is to predict the mass-loss rate of a fire ignited on a vertical surface, given the dimensions of the pyrolyzing zone, some material parameters corresponding to the wall, any external heat fluxes, and some properties of the flame and plume. Although much of the physics involved in burning is well understood, the complexity of the processes is such, especially for turbulent diffusion flames in an enclosure, that modeling all aspects of it from first principles is as yet an impossible task. The $k-\epsilon-g$ approximation (which does not purport to describe the soot-formation process), has had some success in describing the flame and its radiation $[8,9]$, but is very complex. It is demonstrated in this paper that by using some we11-known relationships plus one new result, the mass-burning rate can nevertheless be fairly accurately predicted, with a quite simple integral model. Moreover, in distinction from earlier work, this is extended to the case where there is strong stratification in the enclosure. The key is the finding that the mean radiation absorption coefficient varies with height, 
and that it does so in a simple way. The present model is limited to yielding the steady-state mass-loss rate of non-charring solids.

\section{THE PYROLYSIS RATE}

The mass-loss rate can be calculated either point by point, or global. 1y. Since we are principally interested in the total mass-loss rate, any calculations which are made point by point - i.e., as a function of height, will be integrated. To begin, we assume that the ignited (and therefore pyrolyzing) region is rectangular, with the width $w$ and height $z_{p}$. The pyrolysis rate, $\dot{m}^{\prime \prime}$, is proportional to the net heating flux at the surface of the wall: if we have incoming radiation flux $\phi_{\text {in }}$ and incoming convective heat flux $\phi_{c}$, then a heat balance at any point of the surface yields

$\dot{\mathrm{m}} " \mathrm{~L}_{\mathrm{y}}=\alpha_{\mathrm{w}} \phi_{1 \mathrm{n}}+\phi_{\mathrm{c}}-\phi_{\mathrm{rr}}+\mathrm{k} \operatorname{grad} \mathrm{T}$

where $\alpha_{w}$ is the gray-body absorption coefficient of the wall material. Here $\phi_{x \rightarrow}$ is the reradiation flux from the surface,

$\phi_{\mathrm{II}}=\alpha_{\mathrm{w}} \sigma \mathrm{T}_{\mathrm{S}}{ }^{4}$

(the surface is at the temperature $\mathrm{T}_{\mathrm{s}}$; note that this is an absolute, rather than net, flux: the radiation from the environment is to be included with any other incoming radiation $f l u x)$. The radiation flux $\phi_{i n}$ is the sum of the flame radiation $\phi_{\mathrm{r} f}$ and that of any external heat fluxes:

$\phi_{i \mathrm{n}}=\phi_{\mathrm{x} \mathbf{1}}+\phi_{\mathrm{\theta xt}}$

The flux $\phi_{e x t}$ explicitly includes the ambient radiation flux $\sigma \mathrm{T}_{\mathrm{a}}{ }^{4}$ and the "feedback" radiation flux from surrounding hot walls and/or hot gases. $k$ grad $T$ is the heat conduction flux into the solid, and $I_{v}$ is the latent heat of gasification (i.e. of evaporation or pyrolysis) of the wall material. In experimental measurements of the heat of gasification, neither $L_{v}$ nor $k$ grad $\mathrm{T}$ are measured separately. Instead, an approximately steady state is achieved; then the energy required to vaporize a gram of material. is that required to raise its temperature from $T_{a}$, its uniform initial temperature, to $\mathrm{T}_{\mathrm{s}}$, the surface temperature during its vaporization, plus its latent heat of vaporization:

$\mathrm{H}_{\mathrm{v}} \equiv \mathrm{L}_{\mathrm{v}}+\int_{\mathrm{T}_{\mathrm{a}}}^{\mathrm{T}_{\mathrm{s}}} \mathrm{c}_{\mathrm{p}}(\mathrm{T}) \mathrm{dT}$

This is its effective heat of vaporization, and equation (1) becomes

$\dot{\mathrm{m}}^{\prime \prime}=\left[\phi_{\mathrm{r}}+\phi_{\mathrm{c}}-\phi_{\mathrm{r} r}\right] / \mathrm{H}_{\mathrm{v}}$

for the steady state, where $\phi_{x} \equiv \alpha_{w} \phi_{i n}$. Note that radiation blocking (see Ref.[17-19]) is ignored. In the sections to follow, the fluxes on the right hand side of Eq. (5) will be calculated.

\section{THE RADTATION FLUX}

The external flux will depend entirely on the particular circumstances, configurations, etc. In a compartment within which there is a burning wall, it would consist of the radiation fluxes from the igniting flame, from the 
hot layer(s), from the hot walls, floor, and ceiling, and from any other fires. In a laboratory, it might be that produced by a radiant panel. only the radiation flux $\phi_{\mathrm{x}}$ from the wall flame will be considered in this section. In order to calculate it, the flame temperature, thickness, and absorption coefficient must be known. As shown in the work of orloff, Modak, and Alpert (ref [20], hereafter referred to as OMA), the thickness of the flame at the height $z$ above the base of the flame, $d(z)$, increases linearly with height up to the top of the pyrolysis region (see Fig. 1):

$d(z)=\lambda z$,

$$
0<z<z_{p}
$$

where $\lambda$ is the (constant) slope of the flame envelope. OMA found that $\lambda \approx 1 / 16$ for their PMMA fire; we shall assume it to be the same for all materials. Thus the flame profile is that of an inverted wedge, up to $z_{p}$. Although the flame width will explicitly be taken into account, it is here assumed that there are no variations in the fluxes or in the mass-loss rate across the pyrolyzing region, as if this were a two-dimensional calculation.

A straightforward calculation gives the radiation flux as a function of height, for the case where the temperature and the emission coefficient of the hot gases, $\kappa$, are uniform and constant. Simpler, however, is to use the approximation used by OMA: they found the radiation flux from the hot flame gases at any point on the contiguous wall surface by using an equivalent rectangular slab of the same width and length, but of thickness

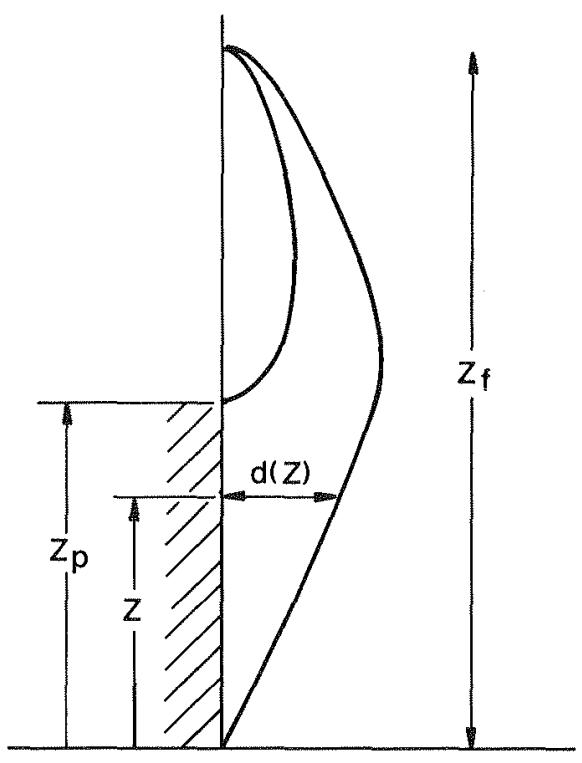

FIGURE 1. Schematic of the appearance of a wall flame. The shaded region is where pyrolysis is taking place.

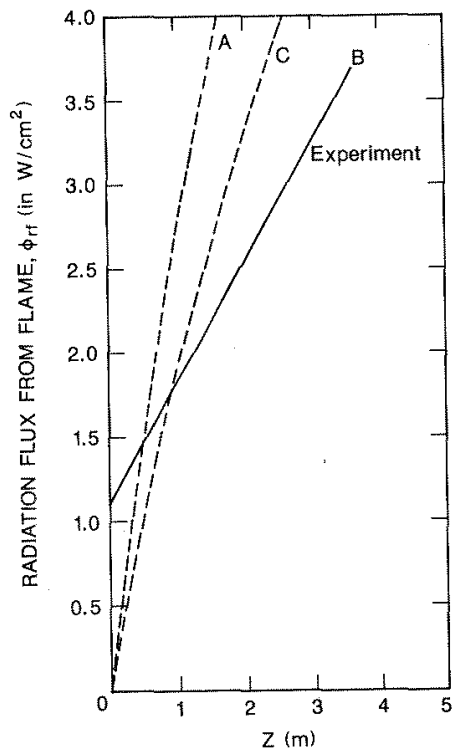

FIGURE 2. Curve $B$ is the radiation flux from the flame, as measured by OMA. A and $C$ result from calculation with a fixed $\kappa$ and two different $\mathrm{T}_{\mathrm{f}}$ 's. 
equal to the local thickness of the wedge. They then used the mean-beamlength approximation for that parallelepiped, for the local flux:

$\phi_{\mathrm{r} f}(z)=\sigma \mathrm{T}_{f}^{4}[1-\exp (-\xi)]$

where $\mathrm{T}_{\mathrm{f}}$ is the (uniform) flame temperature, and $\xi$ is given by

$\xi(z)=3.6 \kappa \mathrm{V} / \mathrm{A}=1.8 \kappa\left[\begin{array}{l}1 \\ \frac{1}{w}\end{array}+\frac{1}{z_{f}}+\frac{1}{d(z)}\right]^{-1}$

where $V$ is the volume of the emitting gas mass and A its surface area. For the wall flame, $w$ is the width of the flame, $z_{f}$ is the flame (and therefore slab) length, and $d(z)$ is the local thickness, given by Eq. (6). This approximation underestimates the flux for $0 \leq z<z_{p} / 2$, and overestimates it for $z_{p} / 2<z \leq z_{p}$. OMA estimated that $\kappa=1.4 \mathrm{~m}^{-1}$ and $T_{f}=1367 \mathrm{k}$, for the PMMA flame; with these values, Eqs. (7) and (8) yield the flux shown by curve $A$ in Fig. 2, On the other hand, their measurements yield a radiation flux which is very well fitted by

$\phi_{x f}=1.10+0.7483 z-0.01 z^{2} \quad W / \mathrm{cm}^{2}, \quad 0.5 \leq z \leq 3.56$

(with $z$ in meters). This is plotted as curve $B$ in the same figure; it is distinctly different from curve $A$. The most important difference is that the value extrapolated to the origin is a significant fraction of the maximum measured value. Choosing a smaller value for $\mathrm{T}_{f}(1250 \mathrm{~K})$ yields curve $C$, with a smaller slope, but does not change the important differences.

However, one need not assume that $\kappa$ is constant; indeed, by working backward from the data, it is possible to infer an effective value for the absorption coefficient and the gas temperature at each height. When this was done, the values shown in Fig. 3 were obtained, and it was found that $T_{f}$ is remarkably constant, at $1285 \mathrm{~K}$. Note, incidentally, that Markstein found a similar variation of $\kappa$ with height for pool fires [21]. Since d(z) is proportional to $z$, it is clear from Eqs. (7) and (8) that as $z$ approaches zero, $\phi_{\text {rf }}$ approaches a value proportional to $z \kappa$. In order to obtain a nonzero value of $\phi_{x}$ at the origin, therefore, the expression for $\kappa(z)$ must approach const. $/ z$. It is remarkable that the absorption coefficient shown in Fig. 3 is in fact very well fitted by a simple binomial, linear in $1 / \mathrm{z}$ :

$\kappa(z)=\kappa_{0}+\kappa_{1} / z \quad\left[\kappa_{0}=0.651 \mathrm{~m}^{-1}, \quad \kappa_{1}=0.562\right]$

This expression is the key which permits one to properly obtain the radiation as a function of height. Note that this is a new application of the experimental results of OMA.

In the simplest approximation, we may take the mean flux to be that received at the midpoint of the slab:

$\bar{\phi}_{\mathrm{r} f} \simeq \sigma \mathrm{T}_{\mathrm{f}}^{4}(1-\exp -\bar{\xi})$

with $\bar{\xi}$ obtained by using Eq. (8), with d given by

$\overline{\mathrm{d}}=\lambda z_{\mathrm{p}} / 2=z_{p} / 32$

$\bar{\kappa}=\kappa\left(z_{p} / 2\right)=\kappa_{0}+2 \kappa_{1} / z_{p}$ 


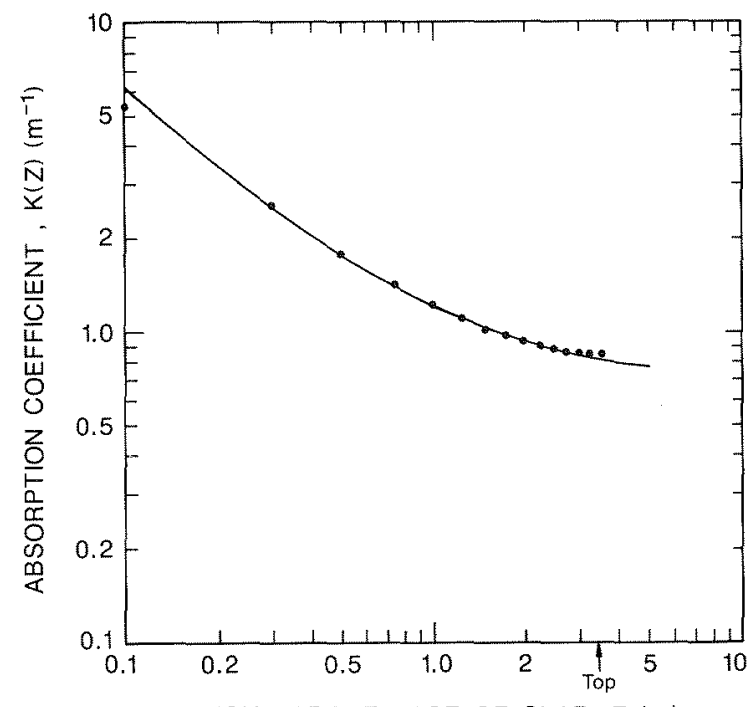

HEIGHT ABOVE BASE OF SLAB, $Z(m)$

FIGURE 3. Absorption coefficient of large PMMA wall flame as a function of height above the base.

According to Eq. $(10), \bar{\kappa}$ is also the volume-weighted average absorption coefficient in the pyrolysis region, $\kappa_{\mathrm{av}}$. Note that $\kappa_{\mathrm{av}}$ is not a constant, so that different-sized slabs will experience different radiation levels. It also follows that $\chi_{\mathrm{R}}$ will not be constant: it also will vary with slab height.

\section{CONVECTION AND RERADIATION}

The mass-loss rate of a burning object whose flame is small and nonluminous is given by the well-known expression [22]

$$
\dot{\mathrm{m}}^{\prime \prime}=\frac{h}{c_{p}} \ln (1+B)
$$

where $B$ is the spalding mass-transfer number, $h$ is the heat-transfer coefficient, and $c_{p}$ is the specific heat of the gas. B can be written as

$\mathrm{B}=\left[\nu \mathrm{y}_{\mathrm{o}} \mathrm{H}_{\mathrm{c}}+\mathrm{c}_{\mathrm{p}}\left(\mathrm{T}_{\mathrm{g}}-\mathrm{T}_{\mathrm{s}}\right)\right] / \mathrm{H}_{\mathrm{v}}$

where $\nu$ is the stoichiometric fuel/oxygen mass ratio, $y_{0}$ is the mass fraction of oxygen in the air, and $\mathrm{T}_{\mathrm{g}}$ is the local gas temperature; the other symbols have already been defined. The first term is the internal energy by which a gram of the air/fuel mixture is incremented upon combustion. When combustion is incomplete, and there is energy loss from the combustion zone via radiation as well, then the appropriate value for the heat of combustion that should be used in Eq. (15) is

$\mathrm{H}_{\mathrm{c}}=\left(\chi_{\mathrm{A}}-\chi_{\mathrm{R}}\right) \mathrm{H}_{\mathrm{c}}$

where $\chi_{A}$ is the combustion efficiency and $\chi_{R}$ the fraction of energy lost to radiation. Moreover, the air-fuel mixture is not stoichiometric in tur- 
bulent diffusion flames, so that instead of $\nu y_{0}=1 / \gamma_{\mathrm{s}}, 1 / \gamma$ should be used, where $\gamma$ is the actual air/fuel mass ratio; note that $\gamma>\gamma_{\mathrm{s}}$. With those changes, $B$ becomes $B^{\prime}$, and is the value to be used in Eq. (14). Note, incidentally, that the first term in Eq. (15) is then approximately $c_{p}\left(T_{f}-T_{g}\right)$. When there is radiation impinging on the pyrolyzing surface, leading to additional heat deposition, there will evidently be an increased rate of pyrolysis. It has been shown $[23,24]$ that for the case where the pyrolyzing gases do not themselves absorb any of the radiation, the radiation can be incorporated as a boundary condition; this permits one to continue to use Eq. (14), but with $B^{\prime}$ further modified by using the "convective B-number"

$\mathrm{B}_{\mathrm{c}}=\frac{\mathrm{B}^{\prime}}{1-\dot{\mathrm{q}}^{\prime \prime} / \dot{\mathrm{m}}^{\prime \prime} \mathrm{H}_{\mathrm{v}}}$

in place of $\mathrm{B}^{\prime}$, where $\dot{q}^{\prime \prime}$ is the net radiative heat transfer flux (including reradiation). From the energy balance equation (5), the denominator in Eq. (17) can be expressed in terms of the convective heat flux; inserting that into Eq.(14), the latter can be rewritten as

$\phi_{c}=\frac{\dot{\mathrm{m}}^{\prime \prime} \mathrm{B}^{\prime} \mathrm{H}_{\mathrm{v}}}{\exp \left(\dot{\mathrm{m}}^{\prime \prime} c_{\mathrm{p}} / \mathrm{h}\right)-1}$

This is the expression used by De Ris [25] and others $[26,27]$. In order to use it, $h / c_{p}$ must be known; from the experiments of Orloff et al [28], the value $h / c_{p}=11.83 \mathrm{~g} / \mathrm{m}^{2} \mathrm{sec}$ can be inferred. This is in fair agreement with the value $13.6 \pm 0.7$ found by Tewarson et al [27] for small horizontal samples.

It may be appropriate to point out here that for a sufficiently small pyrolysis rate $\dot{\mathrm{m}}^{\prime \prime}$, flaming combustion will no longer be sustained. That is a separate issue, however.

If the material is a pure substance (such as water), then there will be a well-defined evaporation temperature $T_{\theta \mathrm{V}}$, and the reradiation flux is given by Eq. (2), with $T_{s} \rightarrow T_{e v}$. For a substance such as PMMA, on the other hand, the (local or global) mass-loss rate depends on the surface temperature; for PMMA it can be expressed as an Arrhenius function:

$\dot{m} "=A \exp \left(-E_{A} / R T\right)$

Therefore (see OMA)

$\phi_{\mathrm{rr}}=\alpha_{\mathrm{w}} \sigma \mathrm{T}_{\mathrm{s} 0}^{4}\left[1-\frac{\mathrm{T}_{\mathrm{so}} \mathrm{R}}{\mathrm{E}_{\mathrm{A}}} \ln \frac{\dot{\mathrm{m}}^{n}}{\dot{\mathrm{m}}_{\mathrm{o}}{ }^{n}}\right]^{-4}$

where $R$ is the universal gas corstant $\left(R=8.314 \mathrm{~J} / \mathrm{mole}{ }^{\circ} \mathrm{C}\right), \mathrm{E}_{\mathrm{A}}$ is the activation energy, $\alpha_{w}$ the grey absorption coefficient, $\dot{m} "$ the actual mass-loss rate, and $m_{0}$ " the mass-loss rate at the reference temperature $\mathrm{T}_{\mathrm{so}}$. (For PMMA, OMA yields $\alpha_{\mathrm{w}}=0.927, \mathrm{~T}_{\mathrm{so}}=636 \mathrm{~K}$, and $\mathrm{E}_{\mathrm{A}}=83.7 \mathrm{~kJ} / \mathrm{mole}$; at $\mathrm{T}=\mathrm{T}_{\mathrm{so}}$, the mass-loss rate is $\dot{\mathrm{m}}_{\mathrm{o}} "=8.316 \mathrm{~g} / \mathrm{m}^{2} \mathrm{sec}$, and $\left.\phi_{\mathrm{rro}}=\alpha_{\mathrm{w}} \sigma \mathrm{T}_{\mathrm{so}}{ }^{4}=8.6 \mathrm{~kW} / \mathrm{m}^{2}\right)$.

\section{CALCULATIONS FOR PMMA}

Measurements made by OMA show that for the $3.56 \mathrm{~m} \mathrm{slab}$, the average mass-loss rate is $\dot{\mathrm{m}}^{\prime \prime}=12.38 \mathrm{~g} / \mathrm{m}^{2} \mathrm{sec}$. The radiation loss fraction has been measured by Tewarson and others [27] to be $\chi_{R}=0.33 \pm .03$ for large PMMA 
TABLE 1. Parameter values chosen for PMMA and its wall flame $[8,20,26]$.

$$
\begin{array}{lll}
\chi_{\mathrm{A}}=0.94 & \chi_{\mathrm{R}}=0.30 & \gamma=10.59 \\
\kappa_{0}=0.651 \mathrm{~m}^{-1} & \kappa_{1}=0.562 & \gamma_{\mathrm{s}}=8.268 \\
\mathrm{H}_{\mathrm{v}}=1.611 \mathrm{~kJ} / \mathrm{g} & \mathrm{H}_{\mathrm{c}}=25.2 \mathrm{~kJ} / \mathrm{g} & \mathrm{h} / \mathrm{c}_{\mathrm{p}}=11.83 \mathrm{~g} / \mathrm{m}^{2} \mathrm{sec} \\
\mathrm{c}_{\mathrm{p}}=1.34 \mathrm{~J} / \mathrm{g}{ }^{\circ} \mathrm{C} & \mathrm{T}_{\mathrm{so}}=636 \mathrm{~K} & \mathrm{E}_{\mathrm{A}}=83.7 \mathrm{~kJ} / \mathrm{mole} \\
\alpha_{\mathrm{w}}=0.927 & \mathrm{~T}_{\mathrm{f}}=1262 \mathrm{~K} &
\end{array}
$$

pool fires; also, $\chi_{\mathrm{A}}=0.94$. Although $\chi_{\mathrm{R}}$ was not measured by oMA for their wall fire, it has been observed [29] that the radiation fraction is generally smaller for wall fires; we have assumed that $\chi_{R}=0.30$. All the parameter values chosen here for PMMA are displayed in Table 1 . Most of these values are taken from the literature, or inferred from the OMA data. The value of $c_{p}$ has been calculated from stoichiometry and knowledge of the specific heats of $\mathrm{O}_{2}, \mathrm{~N}_{2}, \mathrm{CO}_{2}$, and $\mathrm{H}_{2} \mathrm{O}$ at elevated temperatures. That for $\gamma$, from energy conservation and the value of $T_{f}$. Using these values, a calculation can be made for the mass-loss rate for any other vertical PMMA sample: Eq. (5), with Eqs. (12) for $\phi_{r f},(18)$ for $\phi_{c}$, and (20) for $\phi_{x r}$, is a transcendental equation for $\mathrm{m}^{\prime \prime}$. A simple method for solving it is to choose an initial value for the mass-loss rate, $\dot{m}_{1} "$, and calculate $\phi_{r f}$, $\phi_{c}$, and $\phi_{r r}$ with $\dot{\mathrm{m}}_{1}$ "; then $\mathrm{Eq}$. (5) yields a new $\dot{\mathrm{m}}^{\prime}$. A second choice is then made for the mass-loss rate on the basis of this first result, and the process repeated. Generally, a dozen iterations suffice to yield an answer to four or five significant figures. $\dot{m}^{\prime \prime}$ is involved in the calculation of $\phi_{r} f$ through the flame height $z_{f}$ in Eq. (8). This height is calculated according to

$z_{f}=0.06\left(\dot{Q}^{\prime}\right)^{2 / 3} \mathrm{~m}$

(see [16]) where $\dot{Q}^{\prime}$ is the total power output of the fire per unit width, in $\mathrm{kW} / \mathrm{m}$. On the other hand, the calculated value of $\dot{m}$ " is insensitive to the exact choice for $z_{f}$; for simplicity one may take $z_{f} \simeq 1.8 z_{p}$ in order to speed the calculation, if desired. Some experiments [5] made earlier than that of OMA used slabs with a width of $41 \mathrm{~cm}$ and height $1.57 \mathrm{~m}$. Proceeding as described above, the value $\dot{\mathrm{m}}^{\prime \prime}=8.92 \mathrm{~g} / \mathrm{m}^{2}$ sec was calculated. This is only 18 larger than the measured value, $8.84 \mathrm{~g} / \mathrm{m}^{2} \mathrm{sec}$. The value calculated for the large slab is $12.46 \mathrm{~g} / \mathrm{m}^{2} \mathrm{sec}$, less than 18 larger than the correct value, $12.38 \mathrm{~g} / \mathrm{m}^{2} \mathrm{sec}$.

\section{COMPARISON WTTH DATA AND DISCUSSTON}

A more challenging test of the algorithm is to see whether it will yield reasonable results fór cases where there is an external flux, and where the local temperatures and oxygen concentrations vary. This is the situation for a wall burning in a compartment. There, indeed, the fluxes, temperatures, and $\mathrm{Y}\left(\mathrm{O}_{2}\right)$ all vary with position. Since the algorithm has been developed for the case of uniform conditions, it must be generalized. The simplest approximation is to use area-weighted values of $Y\left(\mathrm{O}_{2}\right)$ and $\mathrm{T}_{\mathrm{B}}$ in Eq. (15), and assume that $\chi_{A}-\chi_{R}$ remains unchanged in Eq. (16). (This is nearly identical to summing the contributions from each zone). In a twozone model, this is particularly easy to do, and that is the procedure used in the following. 
Steckler [30] has carried out a series of wall-fire burns on a reduced scale; the floor of the compartment was $1 \mathrm{~m} \mathrm{x} 1 \mathrm{~m}$, and it was $75 \mathrm{~cm}$ high; a doorway $55 \mathrm{~cm}$ high provided the only ventilation. A 1"-thick slab of PMMA, $28.25^{\prime \prime}$ high and $7.31 "=18.57 \mathrm{~cm}$ wide was attached to the rear wall. A line burner ignited the PMMA; when the latter was fully involved, the burner was turned off, and the fire allowed to develop. This development was sufficiently slow that the quasi-steady approximation is probably valid throughout most of the experiment. Sensors measured the various temperatures, fluxes, and oxygen concentrations. A load cell measured the mass of the slab, and therefore the mass-loss rate could be calculated. That is shown, for his run \#9 (with the doorway width at $33 \mathrm{~cm}$ ), by the solid curve in Fig. 4 . The algorithm described above was used to calculate $\dot{m}^{\prime \prime}$ (and therefore $\dot{m}$ ) at several times; those results are shown by the open circles, which have been joined by a smooth dashed curve. It is seen that the agreement is quite good from $t=468 \mathrm{sec}$ (when the igniting flame, which gives an extra, unmeasured, flux, was extinguished) to $t \simeq 1300 \mathrm{sec}$. It is increasingly poor from there to $t \simeq 1500 \mathrm{sec}$; after that time, comparisons cease to be meaningful, as the slab buckled and collapsed starting at that point.

The agreement with the steckler experiment for $468 \leq t \leq 1309$ is surprisingly good, in view of the crudity of the approximations. On the other hand, one may ask why then there is a significant disagreement in

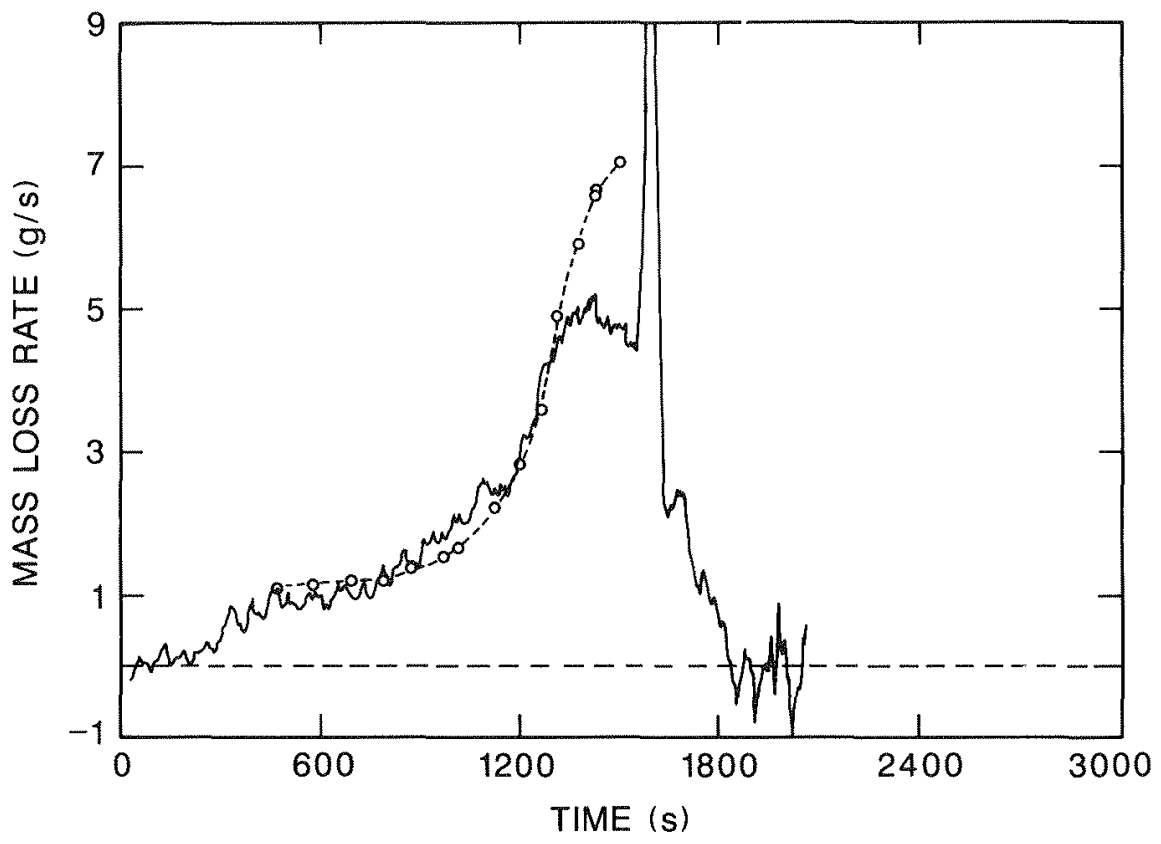

FIGURE 4. Mass-1oss rate of PMMA slab in steckler's experiment number 9 (with a vent width of $33 \mathrm{~cm}$ ). The dashed curve shows the calculated values. 
the region $t>1309$. There are five possible explanations for this disagreement:

1. Radiation blocking, which has been ignored, will reduce the flux absorbed by the PMMA, and the effect will be strongest at high mass-loss rates.

2. The calculation assumes that a steady state existed, whereas the experimental conditions were transient.

3. The PMMA melts, as well as pyrolyzing, and this will

a) uncover lower-temperature material in the upper regions, and

b) cover (and thereby inhibit the pyrolysis of) material in the lower regions.

4. The radiation from a flame burning with vitiated air is weaker than that from the usual flame.

5. The oxygen concentration in the upper layer may be too low to support combustion there, in the late stages ("oxygen starvation").

These phenomena are subjects for future investigations. Note, however, that the contribution to the disagreement from items $d$ and e must be small in this case, since the data shows that [according to Eq.(5)], the external flux alone, without any contributions from the flame, should still yield a mass-loss rate in excess of the measured value, for $t>1350$.

The method described in this paper is easy to use, and is quite accurate for PMMA. However, it requires extensive knowledge of material and flame properties. For the flame emission coefficient, indeed, it requires information $\left(\kappa_{0}, \kappa_{1}\right)$ which is not generally available. This may be viewed as an experimental challenge; in order to obtain results immediately, one can speculate that since $\kappa=1.5 \mathrm{~m}^{-1}$ when it is measured for a pool fire [31],

$$
\kappa_{0} \simeq 0.651 \kappa / 1.5=0.434 \kappa \quad \text { and } \quad \kappa_{1} \simeq 0.562 \kappa / 1.5=0.375 \kappa \text {. }
$$

Accurate measurements of $\chi_{R}$ for two slabs of (very) different sizes can be used to infer $\kappa_{0}$ and $\kappa_{1}$.

An alternative calculation which has been examined is to use an integral (global) approach, using $\chi_{\mathrm{R}}$ in the pyrolyzing region and that over. it, rather than $\kappa(z)$. However, that presents an analogous difficulty: it requires that one know what fraction of the total energy released falls on the pyrolyzing portion of the slab; indeed, it requires knowing it very accurately, as the calculation is very sensitive to that fraction. For the $3.56 \mathrm{~m}$ case, this fraction is 8.228 ; this is just as unknown a number, generally, as are $\kappa_{0}$ and $\kappa_{1}$. Moreover, it is not constant: for the 1.57meter slab, the figure is 7.458 .

An interesting question is the sensitivity of the results to the parameter values which are chosen. For example, suppose that $\chi_{R}$ had been taken to be $\chi_{R}=0.26$ rather than 0.30 , above. In order to get the same value of $\phi_{o}$ for the large slab, $h / c_{p}$ would then have to be chosen to be 0.058 larger than found above, and the mass-loss rate calculated for the smaller slab would then be ${ }^{0}$.05\% greater than calculated above. Thus, the results are insensitive to the precise value chosen for $\chi_{R}$. 


\section{REFERENCES}

1. MacArthur, C.O., and Reeves, J.S., "Dayton Aircraft Cabin Fire Mode1", Report No. FAA-RD-76-120 (1976).

2. Chung, G., Siu, N., and Apostolakis, G., "COMPBURN II: Code Description and Simulation of Experiments, " UCLA-ENG- 8404 (1984).

3. Mitler, H.E., Fire Safety Journal 9 (1985) No. 1-2, p.7

4. Smith, E.E., and Satija, S., J. Heat Transfer, 105 (1983) 281.

5. Jones, W.W., Fire Safety Journal 9 (1985) No. 1-2, p.55.

6. Paxikh, J.S., et al, "Survey of the State of the Art of Mathematical Fire Modeling," U.L. report, file NC 554 (1983).

7. Delichatsios, M.A., 21st symposium (Int.) on Combustion, The Combustion Institute, Pittsburgh, PA (1987) p. 53.

8. Tamanini, F., 17th International Symposium on Combustion, The Combustion Institute, Pittsburgh, PA. (1979) p.1075.

9. Ahmad, T., and Faeth, G.M., 17th Symp. (International) on Combustion, The Combustion Institute, Pittsburgh, PA. (1979) p.1149.

10. Sibulkin, M., and Kim, J., Comb. Sci. and Technology 17(1977) 39.

11. Fernandez-Pe1lo, A.C., Comb. Sci. and Technology $17(1977) 81$.

12. Quintiere, J.G., Fire Safety J. 3(1981)201.

13. Delichatsios, M.A., "Flame spread on a Vertical Wall With a Constant External Heat Flux", presented at the Eastern Section of the Combustion Institute, Nov. 1981.

14. Saito, K., Quintiere, J.G., and Williams, F.A., Fire Safety Science Proceedings of the First International Symposium (Eds., C.E. Grant and P.J. Pagni); Hemisphere Publishing Corp. (1985) p.75.

15. Quintiere, J.G., Harkleroad, M., and Hasemi, Y., Comb. Sci. and Tech. $48(1986) 191$.

16. Hasemi, $Y .$, in Fire Safety Science (Proceedings of the First Int'I Symposium), Eds. C.E. Grant and P.J. Pagni, Hemisphere Publishing Corp.(1986) p.87

17. Gardon, R., "Thermal Radiation", Ph.D. Thesis, U. of London, 1959.

18. Kashiwagi, T., Comb. and Flame 34(1979) 231.

19. Kashiwagt, T., Comb. Sci. and Tech. 20(1979) 225 .

20. Orloff, L., Modak, A.T., and Alpert, R.L., 16th (Int'1) Symposium on Combustion; the Combustion Institute, Pittsburgh, PA. (1977) p.1345.

21. Markstein, G.H, 18th (International) Symp, on Combustion; the Combustion Institute, Pittsburgh, PA. (1981) p. 537.

22. Spalding, D.B., 4th (International) Symposium on Combustion; the Combustion Institute, Pittsburgh, PA., (1953) p.847.

23. Fineman, S., M.S.E. Thesis, Dept. Aero. Eng., Princeton Univ., Princeton, New Jersey, 1962.

24. Glassman, I., Combustion, Academic Press, New York, 1977.

25. De Ris, J., 17th (International) Symposium on Combustion; the Combustion Institute, Pittsburgh, PA.(1979) p.1003.

26. Tewarson, A., "Physico-Chemical and Combustion/Pyrolysis Properties of Polymeric Materials", NBS-GCR-80-295 (1980).

27. Tewarson, A., Lee, J.L., and Pion, R., 18th (Internationa1) Symposium on Combustion; the Combustion Institute, (1980) p.563.

28. Orloff, L., De Ris, J., and Markstein, G.H., 15th (Int'1) Symposium on Combustion; the Combustion Institute, Pittsburgh, PA (1975) p.183.

29. Markstein, G.H., "Radiant Emission from Wall. Fires," Fal1 Technical Meeting, Eastern Section, the Combustion Institute; Philadelphia, PA., Nov, $4-6,1985$.

30. Steckler, K.D., National Bureau of Standards, private communication, 1987.

31. Markstein, G.H., 17th International Symposium on Combustion; the Combustion Institute, Pittsburgh, PA (1978) p.1053. 